recently with hypophosphatasia who is receiving pioneering enzyme replacement treatment.

Baby I was born at 34/40 and required ventilatory support from birth due to respiratory insufficiency. She was noted to have short limbs, hypotonia, and thin ribs on x-rays. Her serum alkaline phosphatase was low; urinary phosphoethanolamine and serum calcium were elevated confirming hypophosphatasia.

In a recent multinational study of 11 patients with severe hypophosphatasia, treatment with recombinant human bone targeted TNSALP (ENB 0040) has been shown to improve bone mineralization. This was associated with healing of rickets, improved developmental milestones and pulmonary function.

Under guidance from the regional Metabolic Bone team at Manchester and with parental consent, Baby I was commenced on ENB 0040 (Asfotase alfa) at the age of 4 weeks with subcutaneous injections three times a week. The drug is being offered to this infant on compassionate grounds by the manufacturer (Alexion pharmaceuticals).

Within 6 weeks of treatment calcium requirement of infant has increased and X-rays have demonstrated remarkable improvement in mineralisation. She remains ventilator dependant with a tracheostomy in situ but, we anticipate that with ENB 0040 treatment, improvement in bone mineralisation and muscle function will facilitate weaning from ventilation.

\section{PF.61 STARRY SKY PATTERN OF FETAL LIVER ASSOCIATED WITH TWIN ANAEMIA POLYCYTHAEMIA SEQUENCE IN MONOCHORIONIC TWINS}

doi:10.1136/archdischild-2013-303966.070

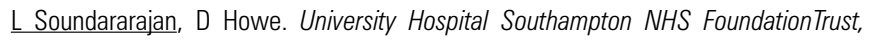
Southampton, UK

Twin anaemia-polycythaemia sequence (TAPS) is an atypical form of twin-twin transfusion syndrome (TTTS) that presents as a large inter-twin haemoglobin difference with one twin developing anaemia and the other developing polycythaemia, without oligohydramnios-polyhydramnios sequence. The prenatal diagnostic criteria for TAPS require that the middle cerebral artery-peak systolic velocity (MCA-PSV) measure greater than 1.5 multiples of median (MoM) in the anaemic twin and less than 0.8 MoM in the polycythaemic twin.

Starry-sky liver appearance was first described in 1980 by Kurtz et al, as corresponding to oedematous swelling of hepatocytes causing decreased echogenicity of the parenchyma (sky) as well as better visualisation of the fibrous walls of the portal vein (stars).

We present a case of monochorionic diamniotic twins with spontaneous twin anaemia- polycythaemia sequence (TAPS) in which the rare sonographic appearance of starry sky liver was seen in the recipient (polycythaemic) twin. The polycythaemic twin had starry sky liver appearance from 25 weeks gestation prior to any sonographic features of TAPS. She was delivered at 28 weeks 6 days gestation when diagnosis of TAPS was made, due to deterioration in growth and Dopplers. One twin was anaemic with haemoglobin of $10.5 \mathrm{~g} / \mathrm{dl}$ and the other polycythaemic with haemoglobin of $28.0 \mathrm{~g} / \mathrm{dl}$.

Conclusion In our case, starry sky appearance of the liver was the early sonographic feature of Twin anaemia polycythaemia sequence.

\section{PF.62 COMPUTATIONAL RECONSTRUCTION OF HUMAN FETAL CARDIAC VENTRICULAR WALL DEVELOPMENT}

doi:10.1136/archdischild-2013-303966.071

'E Pervolaraki, ${ }^{2} \mathrm{R}$ Anderson, ${ }^{3} \mathrm{M}$ Paley, ${ }^{1} \mathrm{AV}$ Holden. 'Faculty of Biological Sciences, University of Leeds, Leeds, UK; ${ }^{2}$ MRC Centre for Reproductive Health, University of Edinburgh, Edinburgh, UK; ${ }^{3}$ Academic Radiology, University of Sheffield, Sheffield, UK
We construct a computational model of the electrophysiology of the developing heart.

Anisotropic geometry at $\sim 100 \mu \mathrm{m}$ voxel resolution was obtained from Diffusion Tensor and Fast Low Angle Shot Magnetic Resonance Imaging and electrical activity from fetal electrocardiograms obtained longitudinally from one gestation and from multiple studies.

Transmural myofibre organisation is established by 136 days gestational age (DGA) and during $2^{\text {nd }}$ and $3^{\text {rd }}$ trimester $\mathrm{OR}$ intervals decrease by $20 \mathrm{~ms}$ while heart dimensions increase by a 2 -fold. This implies an increase in the ventricular conduction velocity. A computational model of the 140 DGA human ventricle is presented that combines cell electrophysiology with anisotropic geometry.

\section{PF.63 A RARE CASE OF FETAL ANAEMIA DUE TO CONGENITAL PYROPOIKILOCYTOSIS TREATED BY INTRAUTERINE FETAL BLOOD TRANSFUSION}

doi:10.1136/archdischild-2013-303966.072

${ }^{1}$ K Lim, ${ }^{1}$ JEAK Bamfo, 'ED Johnstone, ${ }^{2} \mathrm{~A}$ Will. ${ }^{1}$ Department of Obstetrics and Gynaecology, St Mary's Hospital, Mancheseter, UK; ${ }^{2}$ Department of Paediatric Haematology, Central Manchester University Hospital, Manchester, UK

We present the first case of a pre-natal diagnosis of fetal anaemia due to congenital pyropoikilocytosis treated with intrauterine fetal blood transfusion.

A 31 year old woman of Caucasian origin was referred to the fetal medicine unit at 29 weeks gestation with suspected fetal anaemia. She had 3 previous miscarriages and delivered a term female infant who was severely jaundiced and anaemic at birth, requiring multiple exchange transfusions. That infant was diagnosed with congenital pyropoikilocytosis.

In this pregnancy, a middle cerebral artery Doppler peak systolic velocity (MCA PSV) performed at 26 and 28 weeks gestation suggested mild to moderate fetal anaemia. At 29 weeks, MCA PSV indicated severe fetal anaemia. There were no signs of hydrops fetalis. Fetal blood sampling confirmed fetal anaemia and fetal blood transfusion was performed. Fetal blood film confirmed congenital pyropoikilocytosis. At 31 weeks, a repeat fetal blood transfusion was indicated but was unsuccessful due to transient fetal bradycardia. Delivery was prompted and at 32 weeks, a female infant was delivered by elective caesarean section. The infant was anaemic requiring multiple exchange transfusions. Neonatal recovery was uneventful.

Congenital pyropoikilocytosis is an autosomal recessive rare hemolytic anaemia due to an erythocyte membrane defect. It is more often seen in black populations and has rarely been seen in white European populations. Doppler prediction of fetal anaemia using MCA PSV should be advocated in women whose previous pregnancies show them to be at high risk of recurrent fetal or neonatal hemolytic anaemias due to rare erythrocyte defects.

\section{PF.64 COMPLICATED SEQUELAE OF PARVOVIRUS AFFECTED PREGNANCIES}

doi:10.1136/archdischild-2013-303966.073

K Flood, N Burke, S Muellers, FD Malone. Royal College of Surgeons in Ireland, Dublin, Ireland

During the recent epidemic of Parvovirus infection, three complicated pregnancies were managed in the Rotunda Hospital. The fetuses were significantly affected in all three cases, presenting with ultrasonographic findings consistent with severe anaemia; all required intra-uterine fetal transfusions.

Case 1: The first case involved a 30 year old multip who presented at 20 weeks with severe fetal hydrops and a history of Parvovirus 
exposure. Severe fetal thrombocytopenia was noted at the time of cordocentesis. Repeated intrauterine transfusions were required however fetal cardiac function deteriorated further which resulted in fetal demise.

Case 2: The second cases involved a 32 year old multip with confirmed Parvovirus infection who was referred with severe fetal hydrops. Severe thrombocytopenia was again noted however a successful fetal transfusion was performed. Unfortunately the mother subsequently developed Ballantyne (Mirror) syndrome which resolved with expectant management.

Case 3: The final case involved a 28 year old multip with a dichorionic twin pair both of which were severely anaemic with similar haematocrit levels at cordocentesis. Both twins received the same treatment course however different outcomes were encountered.

This case series demonstrates the various complications that add further challenging features to the management of pregnancies affected by Parvovirus infection.

\section{PF.65 WITHDRAWN BY AUTHOR}

\section{PF.66 PROGNOSTIC SIGNIFICANCE AN ENLARGED FETAL STOMACH IN THE SECOND TRIMESTER}

doi:10.1136/archdischild-2013-303966.074

J Richardson, S Boxall, J Stanwell, D Wellesley, K Brackley. University Hospital Southampton NHS Foundation Trust, Southampton, UK

Introduction Duodenal atresia classically presents with a "double bubble" sign and polyhydramnios in the third trimester. The significance of an enlarged stomach detected on a second trimester scan is unclear.

Methods A retrospective review of cases identified from the Wessex Fetal Medicine and Antenatally Detected Anomaly (WANDA) regional databases from 1995 to 2012. Scan reports were reviewed and correlated with outcome.

Results 33 cases of an enlarged stomach in the second trimester were identified. In nine there were additional major anomalies: four with gastroschisis, three with cardiac anomalies (including two trisomies), one severe growth restriction with dilated bowel loops and one with renal cystic dysplasia. In the 24 fetuses without additional major anomalies, five had early signs of a "double bubble" with the first part of the duodenum visible. Three $(60 \%)$ had confirmed duodenal atresia after delivery, one with VATER syndrome and one with trisomy 21 . In the 19 cases without an early "double bubble" sign, stomach enlargement resolved in eight (42\%) and persisted in $11(58 \%)$, one with polyhydramnios. In these 19 babies there was one neonatal death following preterm labour at 26 weeks post amniocentesis. In the 18 cases with postnatal follow up, there were no gastro-intestinal anomalies or feeding problems detected.

Conclusions The finding of an isolated enlarged stomach in the second trimester appears to have a good outcome with no associated feeding problems. However if an early "double bubble" sign is seen there is a significant risk of an underlying duodenal atresia.

\section{PF.67 THE INCREASED INCIDENCE OF ECHOGENIC LUNG LESIONS - AN 18-YEAR REVIEW FROM THE WESSEX REGION}

doi:10.1136/archdischild-2013-303966.075

'LJ Stocker, ${ }^{2} \mathrm{D}$ Wellesley, ${ }^{2} \mathrm{R}$ Parasuraman, ${ }^{2} \mathrm{DT}$ Howe. 'University of Southampton, Southampton, UK; 'University Hospitals Southampton NHS Trust, Southampton, UK

Introduction Echogenic lung lesions (ELL) are a heterogeneous group of lung abnormalities that display a variety of features and are inherently difficult to diagnose and characterise antenatally. Included in this group are congenital cystic adenomatoid malformations (CCAM), pulmonary sequestration, broncho-pulmonary atresia, and congenital emphysema.
Objectives To investigate the changing incidence of echogenic lung lesions detected both antenatally and postnatally.

Methods A retrospective review of cases identified from the Fetal Medicine database and the Wessex Antenatally Detected Anomalies (WANDA) congenital anomalies register from 1994 to 2011.

Results We identified a total of 111 cases of ELL in 492,559 births during the stated period. All but six cases were identified on antenatal ultrasound.

In 1994, the total incidence of ELL was 0.37 per 10000 births. By 2011 this had risen to 5.39 per 10000 births, with a progressive incline during the intervening years.

Conclusion We found a nearly 15-fold increase in the incidence of ELL found antenatally in the Wessex region between 1994 and 2011. It is unclear whether this is due to a true rise in the incidence of this abnormality, or due to increased antenatal recognition as a result of improved ultrasound resolution and operator experience.

\section{PF.68 TORCH SCREENING, WHERE ARE WE NOW?}

doi:10.1136/archdischild-2013-303966.076

'SM Halawa, 'L McDermott, 'ML Denbow, 1,2M Donati. 'United Hospitals Bristol NHS trust, Bristol, UK; ${ }^{2}$ Public Health Laboratory, Bristol, UK

TORCH screening is used in pregnancy in a wide number of indications. In 1990 the Public Health Laboratory service advised that individual serology tests, rather than a TORCH screen, should be performed depending upon clinical circumstances) $)^{12}$. Our group confirmed these findings for fetal medicine indications. ${ }^{1}$ The aim of this retrospective study was to determine our progress with the use of TORCH in pregnancy.

Methods A retrospective review of all TORCH tests requested in $\mathrm{St}$ Michael's Hospital in obstetrics and gynaecology between 01/10/2006 and 31/01/2012 was undertaken via the pathology database.

Results 742 tests were undertaken over the study period. 40 indications were identified. There were 4 positive tests for CMV (1\%), with no cases of confirmed toxoplasmosis or rubella. CMV was found in late miscarriage, recurrent miscarriage and multiple fetal abnormality.

Conclusions The incidence of toxoplasma in the UK is 1-2 infections per 1000 pregnancies $^{3}$ and is normally associated with a maternal illness. Rubella is screened for as part of the routine antenatal screen. Our findings have further confirmed the targeted approach to serology screening ${ }^{4}$. We therefore now only perform CMV serology unless there is an overwhelming clinical indication for the addition of toxoplasma testing.

\section{REFERENCES}

1. Abdel-Fattah SA, Bhat A, lllanes S, et al. TORCH test for fetal medicine indications; only CMV is necesary in the UK. Prenat Diagn 2005;25:1028-31.

2. PHLS. TORCH screening reassessed: the laboratory investigation of congenital, perinatal and neonatal infection. 1. UK: Public Health Laboratory Service, 1990.

3. Allain JP, Palmer CR, Pearson G. Epidemiological study of latent and recent infection by Toxoplasmosis Gondii in pregnant women in a regional population in the UK. J Infect 1998;36:189-96.

4. Fayyaz H, Rafi J. TORCH screening in polyhydramnios: an observational study. J Matern Fetal Neonatal Med 2012;25:1069-72

\section{PF.69 IRELAND'S NATIONAL PERINATAL NEUROSURGICAL CLINIC: REFERRAL PATTERNS AND OUTCOMES, 2010-2012}

doi:10.1136/archdischild-2013-303966.077

${ }^{1} \mathrm{CA}$ Walsh, ${ }^{1,4} \mathrm{FM}$ McAuliffe, ${ }^{2} \mathrm{D}$ Crimmins, ${ }^{3} \mathrm{E}$ Laffan, ${ }^{3} \mathrm{~V}$ Donoghue, 'S Higgins, ${ }^{1} \mathrm{R}$ Mahony, 'S Carroll, 'P McParland. 'Department of Fetal Medicine, National Maternity Hospital, Dublin, Ireland; 'Department of Paediatric Neurosurgery, Children's University Hospital, Dublin, Ireland; 'Department of Paediatric Radiology, National Maternity Hospital, Dublin, Ireland; ' $U C D$ School of Medicine and Medical Sciences, Dublin, Ireland 